\title{
Chiprovtsi-Pirot hand-woven carpets from NW Bulgaria and Serbia: symmetrological characteristics of the ornaments
}

\author{
Radostina Atanassova, Rossitsa Vassileva \\ Geological Institute, Bulgarian Academy of Sciences, Acad. G. Bonchev Str., Bl. 24, 1113 Sofia, Bulgaria, \\ e-mail: radi@geology.bas.bg; rosivas@geology.bas.bg.
}

(Accepted in revised form: October 2017)

\begin{abstract}
The man-made periodical patterns executed in textile are especially suitable for the study of plane groups. The diversity of geometric patterns can be described based on the symmetry classification of the twodimensional symmetry, anti-symmetry and colored symmetry.

The symmetrological peculiarities of representative models of hand-woven Chiprovtsi-Pirot (NW Bulgaria and Serbia) carpets have been studied. They share the same elements and ornamental motifs, methods of composition, color combinations, etc. The traditional carpet ornamentations of the Chiprovtsi region are symmetrically organized, with highly stylizing geometric forms. Three different symmetries of the characteristic triangular forms of the ornaments have been recognized: trigonal, hexagonal and tetragonal. The description of their ornamentation revealed motifs as original features, as well as the effects from outside Chiprovtsi=Pirot area. The influence of materials and technology on the resulting pattern symmetry should be carefully emphasized in analysis of the visual art.
\end{abstract}

Atanassova, R., Vassileva, R. 2017. Chiprovtsi-Pirot hand-woven carpets from NW Bulgaria and Serbia: symmetrological characteristics of the ornaments. Geologica Balcanica 46 (2), $27-31$.

Keywords: symmetry, plane groups, two-sided carpets, cultural heritage, Chiprovtsi, NW Bulgaria.

\section{INTRODUCTION}

For many years, scientists have utilized the concept of symmetry to describe both general aspects of the organization of cultural behavior and specific aspects of the structural organization of designs. The clarity of symmetrological definitions is advantageous when compared to many other fields of analysis of visual art (Makovicky, 1986). During the last century, a number of investigators in different disciplines rediscovered symmetry analysis, both as a classificatory tool and as an aid in the discovery of consistencies and differences in cultural activities (Washburn, 1986). The manmade two-dimensional periodical patterns executed in textile are one of the earliest important considerations of symmetry in material cultural objects.

A significant part of the Bulgarian national heritage is the famous hand-woven Chiprovtsi (NW Bulgaria) carpets and tapestries. They are a phenomenon in the Bulgarian folk artistic crafts, prosperous in the Renaissance, existing even nowadays. The first flat two-sided carpets were made in Bulgaria at the end of the $17^{\text {th }}$ and the beginning of the $18^{\text {th }}$ century. Most of the historians and ethnographers consider that carpet weaving on the Balkans, and especially in Bulgaria, originates from Asia Minor. In particular, Ottoman carpet weaving had its greatest breakthrough with prayer carpet manufacturing (Okumura, 2011). Prayer carpets that exhibit regional characteristics became widespread after the $17^{\text {th }}$ century.

The Chiprovtsi carpets do not represent copies (reproduction) of foreign samples, which is typical for the production of Persian carpets. They result from an original recreation, representing the national appearance and the development of ornamentation and color richness and are determined by the Bulgarian national lifestyle, customs, traditions (Stankov, 1960).

Recently, the tradition of carpet-making in Chiprovtsi was selected for inscription on the UNESCO Representative List of the Intangible Cultural Heritage of Humanity (UNESCO Representative List, 2014). The intangible cultural heritage, transmitted from generation to generation, is constantly recreated by communities in response to their environment, their interaction with nature and their history, and provides them with a sense of identity and continuity, thus pro- 
moting respect for cultural diversity and human creativity (UNESCO Convention, 2003).

\section{HISTORICAL NOTES}

The town of Chiprovtsi (Kiprovets, Chiprovets) is rich in history and was also famous all over Europe for its school of goldsmiths. In the $16^{\text {th }}$ and $17^{\text {th }}$ centuries, Kiprovets flourished as a dynamic center of political, economic and cultural life. It all began with active mining of the numerous old silver pits. The high quality of the ore and the silver obtained from it were rated as a special commodity on the Venetian exchange and was in high demand all over the Balkan Peninsula (Sotirov, 1984).

The earliest data about hand-woven carpets in the region date back to the $17^{\text {th }}$ century. From travelers' notes, we understand that carpets were produced in four production centers: Pirot, Chiprovtsi, Samokov and Kotel. Due to the considerable similarity of both the technology and the development of the ornamental composition, the first three belong to one group of West Bulgarian carpets (Stankov, 1960).

The western traveler Ami Boué, who visited the Chiprovtsi region in 1836-1838, reported that "mainly young girls, under shelters or in corridors, engage in carpet weaving". The people of the region have preserved their old-fashioned vertical handlooms and the use of interlacing method to produce flat, two-sided tapestries for floor covering. During the centuries, carpet weaving has been deeply integrated in the social and cultural life of the population of the region (Minkin, 1989).

\section{ORNAMENTATION AND MOTIFS}

The Chiprovtsi-Pirot carpets have specific ornamental composition, motifs and colors. Characteristic for the late compositions is a rim orbiting a square or rectangular field consisting of one or more strips (Fig. 1).

The setup of decoration elements in a carpet composition represents its ornamentation. There was an effort to distinguish the purely geometrical ornamentation from nature-like style not only in the carpet weaving, but also in the ceramics and pottery, woodcarving, metal products, etc. The use of these two styles was subject not only of esthetic preferences but strongly depends of the technical performance as well. Reproducing curves, ovals, leaves, animals and other figures in the carpet weaving is impossible, unless the fibers are very thin, as in silk Persian carpets. The decoration in the two-sided flat carpets is styled on the basis of mainly squares and triangles (Fig. 2), which does not evidence for geometrical preferences. The first ornamental form determined by this weaving technique is the triangle, and this was recognized from exhaustive early work of Stankov (1960).

The weaving technique itself determines the triangular, square or rhombic-like shapes, including interlace between stretched parallel threads (base) of other threads called weft. Differently colored threads were used for the weft and a different pattern was obtained.

Their original color schemes often represent either dichroic or multicolored plane groups (Atanassova and Vassileva, 2016). Some motifs are closely interlocked and, although recognizable as various figures, they simply function as motifs in the pattern, in some cases structured by complex two-, three- or four-fold symmetries (Fig. 3).

Names such as "karakachka," "makaz," "kanatitsa" are terms that weavers use among themselves to indicate different motifs. Chiprovtsi-Pirot carpets often displayed compositions like "bakam," "garibaldi," "esenna loza," "saksiykite," "kufarite," "tree of life," etc. The "tree of life," which appears as symmetrically $\mathrm{V}$-shaped towards the periodicity direction ornaments (Fig. 3b), is a motif frequently used also in Anatolian

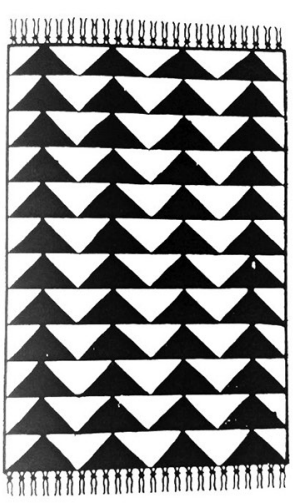

246

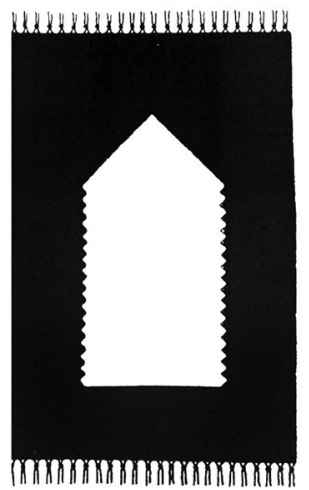

251

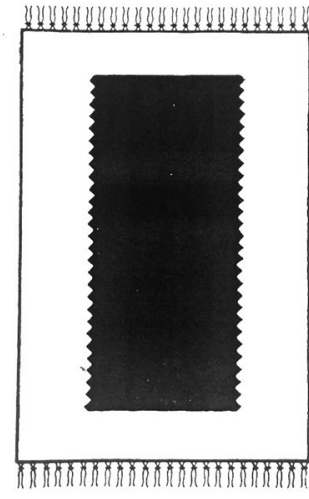

255

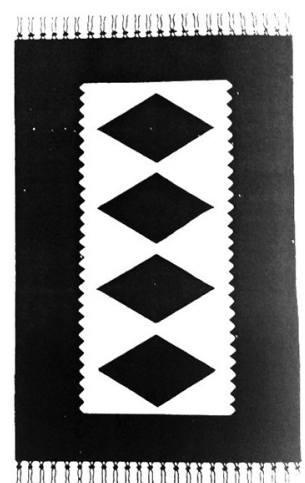

258

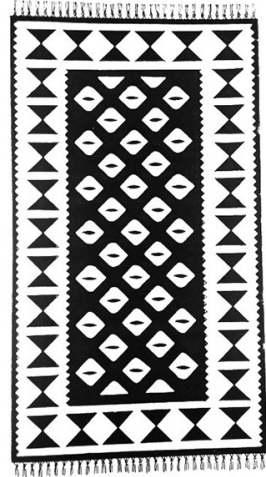

235

Fig. 1. Various representative carpet compositions (sample numbers after Stankov, 1960). 

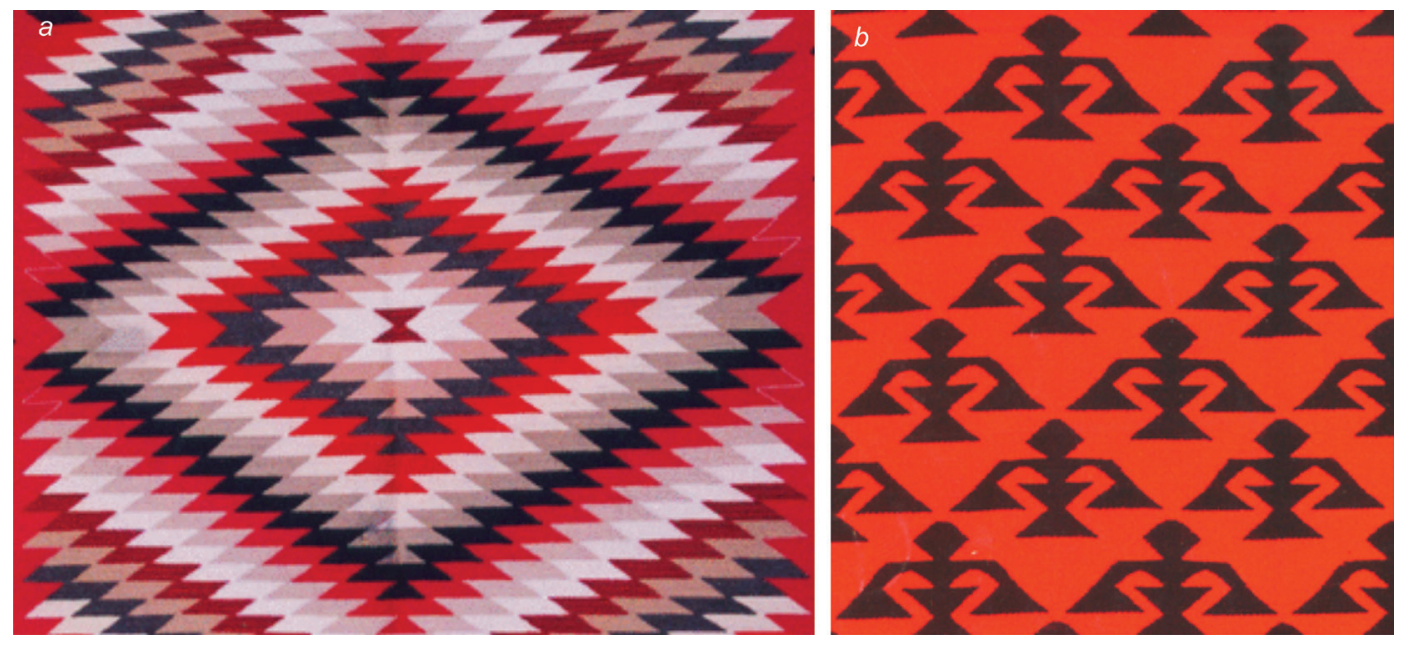

Fig. 2. Details of carpet models: $a$ ) "kolo" with symmetry pmm with pseudorhombic shape; $b$ ) "karakachka" with motifs with symmetry $p 4$.

kilims (Ünal, 2009). Some authors believe that it symbolizes the uniqueness of God, paradise and immortality. It is generally depicted with birds on it.

Some carpet models from Pirot are distinguishable by their density of composition, which is often obtained by uniform filling with the same ornamental motifs. This peculiarity is similar to the distinguishing features of the embroidery from the lands to the west of present-day Bulgaria (Gutseva, 2017).

Interesting fact is that the motif "kanatitsa," the most distributed in carpet models, is regularly used as well as in internal and external architectural deco- rations of different parts of local public and private buildings in the region of Chiprovtsi.

\section{SYMMETROLOGICAL CHARACTERISTICS}

It is well known that there are 17 classes of symmetry groups of planar ornaments (Fig. 4), which repeat in at least two non-parallel directions (Shubnikov and Koptsik, 2004). Concerning the symmetry elements and operations in different carpets from Chiprovtsi and Pirot, there have been recognized the $p 1, p m, \mathrm{~cm}$,
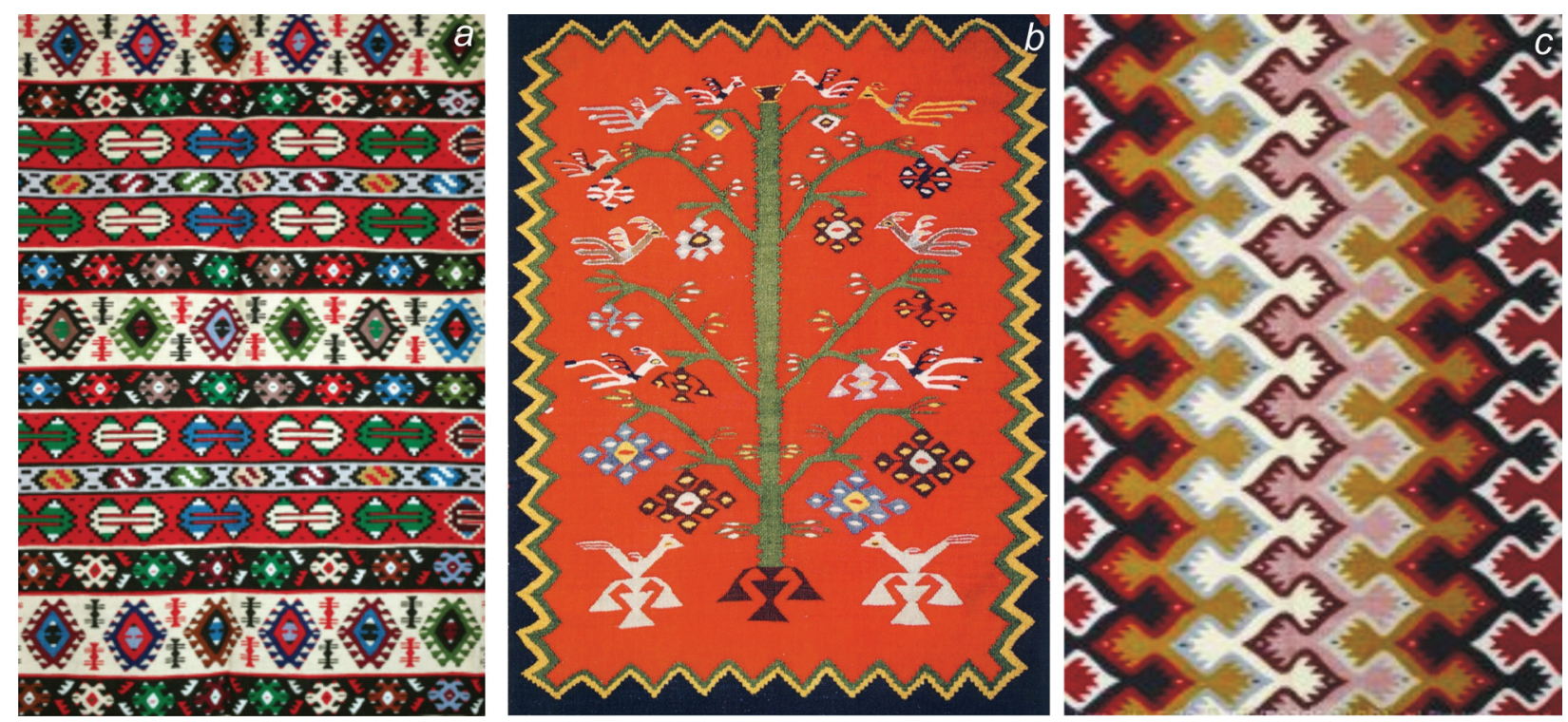

Fig. 3. Exhibited samples in the Chiprovtsi museum collection: $a$ ) a detail of the model "kufarite," $20^{\text {th }}$ century; $b$ ) "tree of life," reproduction from the carpet model; $c$ ) a detail from Pirot carpet with densely filled composition with motif "kostenitsa." 
$p 2 m m, p 2 m g, p 4, p 4 m m$ plane groups and some other arrangements. Approximate analysis can be made on the symmetry of the ornaments and motifs used in textile, while the spatial relation of its individual elements to the elements of the whole symmetry is uncertain. Frequently, the placing of asymmetric elements (ornaments) on symmetry operators reduces the total symmetry.

When a set of same motifs is denoted by color, the geometrical pattern becomes a color pattern. This color pattern illustrates a group that is a subgroup of the group of the pattern; this subgroup determines, or is determined by, the ways in which the colors are permuted by geometrical symmetry operations (Senechal, 1975). In such manner, some late models from the ornamental period have received and formed an exceptionally rich decorated style (Fig. $3 a$ ).

Twinning phenomenon, as in crystalline nature, was used from the Chiprovtsi and Pirot masters, and the beauty of the principle was used in the complex models. Characteristic with such arrangements is the model "kufarite" from the ornamental period from the $20^{\text {th }}$ century (Fig. $3 a$ ).

Adding of black-and-white (anti-)symmetry to plane groups led to the design expressivity of the oldest carpet examples (Fig. 1), exposed nowadays in the museum of Chiprovtsi.

\section{DISCUSSION}

It has been observed that most designs produced by cultures throughout the world are symmetric, and the designs in any given culture are organized by just a few symmetries, rather than by all classes of the plane pattern symmetries (17 two-dimensional classes). Washburn (1986) mentioned that we do not yet understand why there is preferred use of several symmetries or how these preferences relate to other activities in culture.

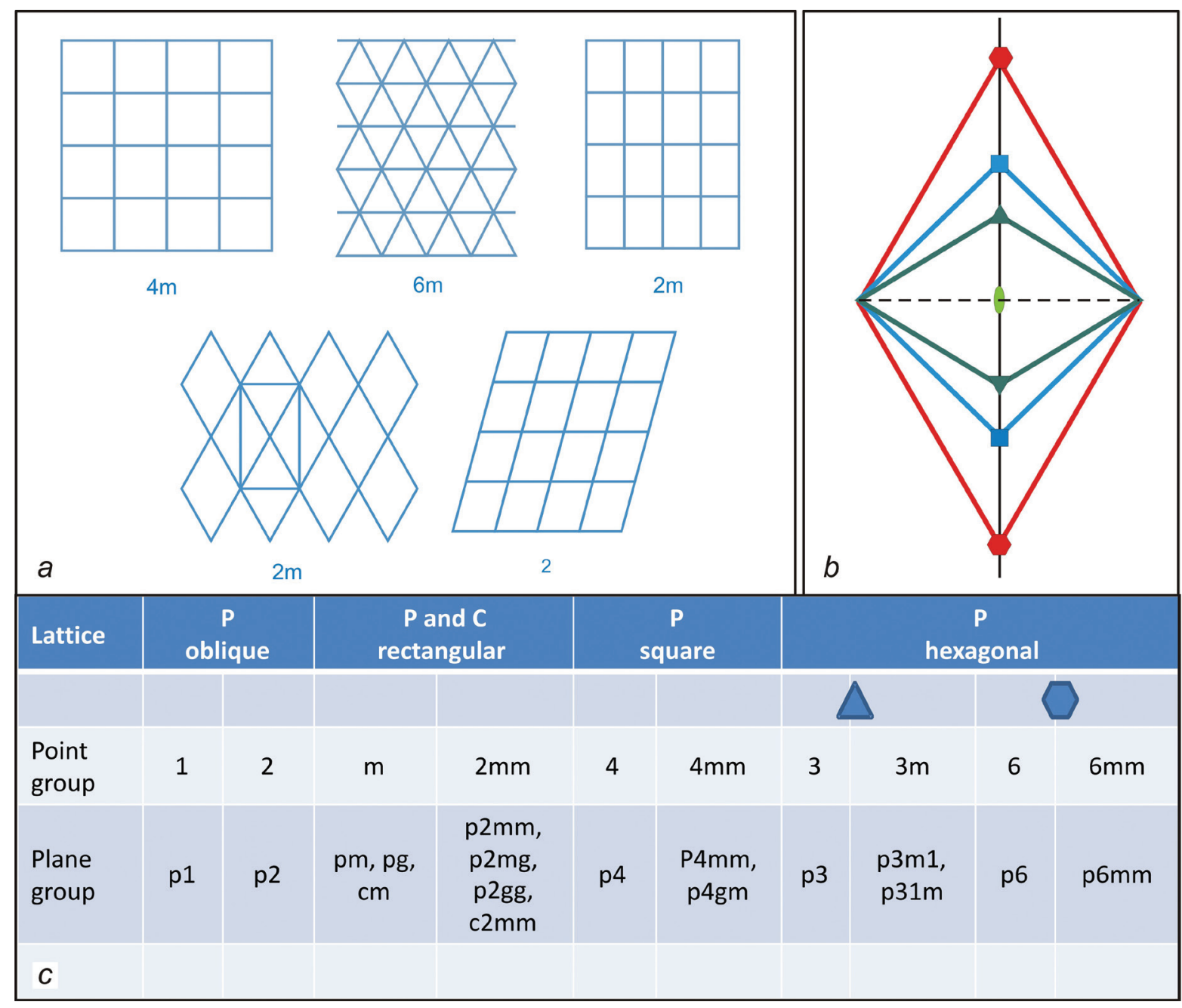

Fig. 4. The plane symmetry groups: $a$ ) five possible plane tilings by regular polygons (lattices), $b$ ) possible symmetries of the ornamental motifs determined from the techniques in the studied carpet models; $c$ ) 17 plane groups formed by the combination of 10 Hermann-Mauguin point groups and 5 type lattices. 
The Arabic geometrical art with its preponderance of hexagonal or trigonal patterns stands unique in the history of ornamental art, while the two-dimensional geometrical patterns of antique Greece and Rome in the great majority were based on orthogonal axial systems (Makovicky and Makovicky, 1977).

We suppose that the influence of material and technology on the resulting pattern symmetry should be considered. As in studied hand-woven flat carpets from the region of Chiprovtsi-Pirot, the weaving technique determines the trigonal, tetragonal or rhombiclike symmetries of the motif shapes (Fig. 4b).

One of the important questions to be understood in the process of symmetrological analysis of a pattern is the spatial relation of its individual elements to the elements of whole symmetry (Makovicky, 1986). The placing of asymmetric elements (ornaments) on symmetry operators reduces the total symmetry; the placing of highly symmetric objects into positions with lower or low local symmetry in the symmetry group leads to the local enhancement of symmetry and potentially also to order-disorder phenomena.

\section{CONCLUSIONS}

Characterization and description of the geometric patterns and ornaments involve more than tools and methodology; they involve the logical process of interdisciplinary research. The most distinctive feature of the Chiprovtsi-Pirot carpets can be outlined as use of simplified forms and stylized models structured by complex symmetries. They share the same ornamental motives, methods of composition, color combinations, etc.

Recognized are three different symmetries of the characteristic triangular forms of the ornaments, which were mentioned already in the early work of Stankov (1960), although without distinguishing between them.

Carpet designs that, when colored, do not have symmetries, identical with their original structural symmetries. That is, the colored design does not preserve the symmetries of the original uncolored design.

In conclusion, we believe that the influence of materials, as well as technology, on the resulting pattern symmetry should be carefully emphasized.

\section{Acknowledgements}

The authors thank both Ilko Dimitrov from Chiprovtsi and the Curator of the Chiprovtsi Museum for their craftwork demonstrations and help during fieldwork. Thanks are also due to Ruslan I. Kostov for constructive review and helpful suggestions, which improved the manuscript.

\section{REFERENCES}

Atanassova, R., Vassileva, R. 2016. Symmetrological review of the ornamental patterns of the Chiprovtsi hand-woven carpets. Journal of Architecture and Art 5, 1-3.

Gutseva, E. 2017. Signs from the embroidery. National Historical Museum, Sofia, 64 pp. (in Bulgarian).

Makovicky, E. 1986. Symmetrology of art: Coloured and generalized symmetries. Computers and Mathematics with Applications 12B (3-4), 949-980.

Makovicky, E., Makovicky, M. 1977. Arabic geometrical patterns - a treasury for crystallographic teaching. Neues Jahrbuch für Mineralogie Monatshefte 2, 58-68.

Minkin, A. 1989. The Chiprovtsi carpet and the international exhibitions. In: Neshev, G. (Ed), Chiprovtsi 1688-1988. Izdatelstvo na Otechestvenia Front, Sofia, 168-215 (in Bulgarian).

Okumura, S. 2011. The significance of yellow dyed warps in Cairene rugs and a group of Ottoman court prayer rugs. Journal of Carpet Flatweaves, Fabrics, Clothing and Embroideries 5, 88-95.

Senechal, M. 1975. Point groups and color symmetry. Zeitschrift für Kristallographie 142, 1-23.
Shubnikov, A.V., Koptsik, V.A. 2004. Symmetry in science and art. Moscow-Ijevsk: Institute of Computer Investigations, 560 pp. (in Russian).

Sotirov, I. 1984. The Chiprovtsi school of goldsmiths. Darzhavno Izdatelstvo "Septemvri", Sofia, 146 pp. (in Bulgarian).

Stankov, 1964. Chiprovtsi carpets. Bulgarian Academy of Science, Sofia, 152 pp. (in Bulgarian).

Ünal, S. 2009. Symbolic meanings and characteristics of Anatolian kilim motifs. The $25^{\text {th }}$ European Crystallographic Meeting, Istanbul, Turkey, Abstracts, 12-13.

UNESCO 2003. Convention for the Safeguarding of the Intangible Cultural Heritage, $14 \mathrm{pp}$.

UNESCO 2014. Representative List. Ninth session. Paris, France. Nomination File NO. 00965 for Inscription on the Representative List of the Intangible Cultural Heritage of Humanity in 2014, 16 pp.

Washburn, D.K. 1986. Pattern symmetry and colored repetition in cultural contexts. Computers and Mathematics with Applications 12B (3-4), 767-781. 